\title{
H subtype vascular endothelial cells in human femoral head: an experimental verification
}

\author{
Fuqiang Gao ${ }^{1 \#}$, Tianli Mao ${ }^{2 \#}$, Qingyu Zhang ${ }^{3 \#}$, Jun Han ${ }^{1 \#}$, Wei Sun ${ }^{1}$, Zirong Li ${ }^{1}$ \\ ${ }^{1}$ Department of Orthopedics, China-Japan Friendship Hospital, National Health and Family Planning Commission of the People's Republic of \\ China, Beijing, China; ${ }^{2}$ Department of Orthopedics, Peking University Third Hospital, Beijing, China; ${ }^{3}$ Department of Orthopedics, Shandong \\ Provincial Hospital Affiliated to Shandong First Medical University, Jinan, China \\ Contributions: (I) Conception and design: F Gao, W Sun, Z Li; (II) Administrative support: T Mao, J Han; (III) Provision of study materials or \\ patients: F Gao, T Mao, J Han, Q Zhang; (IV) Collection and assembly of data: J Han, Q Zhang; (V) Data analysis and interpretation: F Gao, J Han, \\ W Sun; (VI) Manuscript writing: All authors; (VII) Final approval of manuscript: All authors. \\ \#These authors contributed equally to this work. \\ Correspondence to: Fuqiang Gao, MD; Wei Sun, MD. Department of Orthopedics, China-Japan Friendship Hospital, Beijing 100029, China. \\ Email: gaofuqiang@bjmu.edu.cn; sun887@163.com.
}

\begin{abstract}
Background: Osteonecrosis of the femoral head (ONFH) is difficult to treat, and leads to an impaired microcirculation of the femoral head and activation of a repair response. The type $\mathrm{H}$ vessels have been proved to mediate the growth of the bone vasculature and couple angiogenesis to osteogenesis. The aim of the study is to investigate the experimental verification of $\mathrm{H}$ subtype vascular endothelial cells (HSVECs) in the human femoral head.
\end{abstract}

Methods: Vascular endothelial cells were isolated from femoral heads of patients who underwent hip replacement. Cells were isolated by the methods of enzymic digestion and density gradient centrifugation, purified by differential adhesion and selective medium. The HSVECs were characterized for localization of endothelial cell markers such as von Willebrand factor (vWF), vascular cell adhesion molecule-1 (VCAM1), CD31 and Endomucin (Emcn) by immunofluorescence staining. Cell morphology was observed by microscopy.

Results: The HSVECs expressing vWF, VCAM-1, CD31 and Emcn were identified by immunofluorescence. After 3-4 days of culture, adherent cells were observed evenly distributed in the culture flasks, and inconsistent in size and shape. Following 7-8 days of culture, the cells formed a monolayer after fusion, arranged in fascicular, whorled, and the growth was attachment-inhibited. Most of the cells appeared short spindle, polygon and cobblestone-like morphologic characteristics.

Conclusions: Here we make an effective experimental verification of HSVECs in the human femoral heads, which will facilitate the study of ONFH, hip osteoporosis and other bone diseases in vitro.

Keywords: Blood vessels; endothelial cells; femur head necrosis

Submitted Jan 08, 2020. Accepted for publication Jun 04, 2020.

doi: 10.21037/apm-20-121

View this article at: http://dx.doi.org/10.21037/apm-20-121

\section{Introduction}

Osteonecrosis of the femoral head (ONFH) is among the most common osteoarthritic diseases worldwide, which are caused by hormones, alcohol abuse, hip trauma, and decompression sickness and so on (1-3). ONFH may occur at any age, but almost $75 \%$ of patients with the disease are between 30 and 60 years of age (4). There are a variety of hypotheses for the pathogenesis of this disease, including microvascular injury, intravascular coagulation, intraosseous high pressure, dyslipidemia, hormonal toxicity of bone cells and osteoporosis (5-8). However, its pathogenesis remains elusive. Previous studies have shown that the damage 
and dysfunction of bone microvascular endothelial cells (BMECs) of the femoral head can cause poor blood supply to the femoral head, and leads to the final bone necrosis in the pathogenesis of ONFH $(9,10)$.

Angiogenesis is of critical importance to osteogenesis and is essential for bone development not only in embryo stage but also in bone remodeling thereafter (11-13). New bone formation and fracture healing require vasculature, without which bone tissue cannot be repaired or rejuvenated $(14,15)$. It has been suggested that the close proximity of vascular and osteoblastic cells, and potential roles of angiogenic blood vessel growth in fracture healing (16). Several studies also showed that the capacity for vascular regeneration is significantly impaired in patients with ONFH due to high blood pressure, hypoxia, and lack of growth factors, which leads to reduce new bone formation and weaken mechanical strength of the femoral head $(10,17)$.

According to the differences of specific cell surface markers, Kusumbe et al. (18) found that there were two subtypes of endothelial cells in the capillaries of mouse metaphysis and endosteum. Type $\mathrm{H}$ vessels were strongly positive for CD31 (also known as platelet endothelial cell adhesion molecule-1) and Endomucin (Emcn) as assessed by immunofluorescence staining, whereas type $\mathrm{L}$ vessels exhibited distinct molecular properties and displayed only weak CD31 staining and lower Emcn expression (18). These type $\mathrm{H}$ vessels have been identified mainly in the metaphysis near the growth plate (19), which are rich with several growth factors related to the survival and proliferation of osteoprogenitors. However, type L vessels are mainly distributed in the diaphysis region, and there are few osteoprogenitors around type $\mathrm{L}$ vessels. $\mathrm{H}$ subtype vascular endothelial cells (HSVECs) secrete the protein Noggin, which maintains the osteoblast and chondrocyte cells that are responsible for bone formation. Moreover, HSVECs secrete vascular endothelial growth factor (VEGF), which supports angiogenesis (18), and have been proved to mediate the growth of the bone vasculature, maintain perivascular osteoprogenitors and osteoblasts, and couple angiogenesis to osteogenesis (20). Accordingly, the proliferation of type $\mathrm{H}$ vessels are positively regulated by the $\alpha$-subunit of hypoxia-inducible factor $1 \alpha(\mathrm{HIF}-1 \alpha)$ and Dll4-Notch signalling pathways (18). The abundance of type $\mathrm{H}$ vessels decreased in aged human and mice, and were associated with bone mass loss obviously (18).

Although the pathogenesis of ONFH remains unclear, many researchers agree that $\mathrm{ONFH}$ is associated with thrombosis in the microvasculature of the femoral head which results in blood supply disruption and hypoxemic injury (10). The BMECs constitute the inner lining of the vascular wall, the damage of which is one of the key factors causing blood clot formation (21). Rebuilding and improving the blood supply to the femoral head is considered to be an effective treatment for ONFH. Ding et al. (22) showed that transplantation of endothelial HIF$1 \alpha$ transgenic bone marrow cells (BMCs) enhanced both angiogenesis and osteogenesis and promotes the repair of necrotic area of ONFH. Kusumbe et al. (18) found that HIF- $1 \alpha$ plays an essential role in controlling type $\mathrm{H}$ vessel formation. Therefore, it is of great significance to use the HSVECs in the study of the etiology, pathogenesis and treatment of ONFH.

In earlier researches, the isolation and culture of BMECs were mostly derived from the bone marrow of iliac bone, and the operations were complicated and immature, with poor repeatability (23-25). Yang et al. (26) from our institution used enzyme digestion, density gradient centrifugation and immunomagnetic beads to separate and culture vascular endothelial cells of human femoral head. In order to simplify the operation process and avoid the damages caused by magnetic beads, Lu et al. (27) from our institution used differential adherence method to remove the fibroblasts, and the endothelial cells were further purified by using complete endothelial cell culture medium containing endothelial cell growth factor and heparin to stimulate the growth of vascular endothelial cells. So far, there is no research on identification and culture of HSVECs in the femoral head.

Based on previous studies, this study improved and simplified some technical details to establish a reliable method to the experimental verification of HSVECs of the human femoral head.

\section{Methods}

The trial was conducted in accordance with the Declaration of Helsinki (as revised in 2013). The study was approved by institutional ethics board of China-Japan Friendship Hospital (No. 2016-BZR-2) and informed consent was taken from all the patients.

\section{Source of specimen}

We prospectively enrolled 24 patients who underwent hip replacement, between February 2017 and May 2017, in a single hospital. The inclusion criteria: patients who were 
diagnosed with femoral neck fracture, intertrochanteric fracture, primary hip osteoarthropathy, developmental dysplasia of the hip, or traumatic osteoarthropathy. The exclusion criteria were the following: patients with hip joint diseases such as ONFH, hip joint tuberculosis, ankylosing spondylitis, rheumatoid arthritis, hemophilia hip joint disease, and tumor around the hip joint.

\section{Main reagents}

Collagenase type I, $0.25 \%$ trypsin-ethylenediaminetetraacetic acid (EDTA), $100 \mu \mathrm{g} / \mathrm{mL}$ streptomycin, $100 \mathrm{U} / \mathrm{mL}$ penicillin, and TritonX-100 were purchased from Solarbio (Beijing, China). Ten percent fetal bovine serum (FBS), phosphate-buffered saline (PBS), and Hanks balanced salt solution (HBSS) were purchased from Thermo Fisher Scientific (New York, USA). Gelatin and Hoechst 33342 were purchased from Sigma-Aldrich (St. Louis, MO, USA). Endothelial cell growth medium (ECGM) containing M199 medium, and low-glucose Dulbecco's modified Eagle's medium (DMEM-LG) were purchased from HyClone (Logan, Utah, USA). Paraformaldehyde and ethanol were purchased from Boster (Wuhan, Hubei province, China). Heparin sodium was used from Xinbai pharmaceutical (Nanjing, Jiangsu province, China). Endothelial cell growth factor (ECGF) was purchased from Sino Biological Inc. (Beijing, China). Rat anti-CD31 antibody, rat antivon Willebrand factor (vWF) antibody, rat anti-VCAM-1 antibody and goat anti-rabbit IgG-FITC antibody were purchased from Bioss Inc. (Beijing, China). Rat antiEndomucin antibody was purchased from Absin Bioscience Inc. (Shanghai, China).

\section{Main equipments}

$\mathrm{CO}_{2}$ incubators were the product of Eppendorf (Hamburg, Germany); clean bench was the product of Shiankelin (Beijing, China); inverted phase contrast microscope was the product of Olympus (Shinjuku-ku, Tokyo, Japan); horizontal centrifuge was the product of Sigmae-Aldrich (St. Louis, MO, USA); water bath was the product of SHELLAB (Cornelius, OR, USA); transmission electron microscope was the product of Hitachi (Tokyo, Japan).

\section{Human tissue preparation}

The samples in this study were obtained from the femoral heads of patients who underwent hip replacement in the
Department of Orthopedics of China-Japan Friendship Hospital. Briefly, the soft tissues and cartilages of the samples were removed under aseptic condition in the operation room, and the cancellous bone of femoral heads were bitten into bone particles with bone rongeur. Subsequently, the bone particles were placed into a $50 \mathrm{~mL}$ centrifugal tube containing $30 \mathrm{~mL}$ DMEM-LG medium, and then taken to the laboratory immediately.

\section{Isolation of cells}

The centrifuge tubes with specimens were repeatedly oscillated for 4-5 minutes, and then the medium and the upper lipid were removed. Bone tissues were washed for three times with HBSS, digested with DMEM medium containing $0.2 \%$ collagenase I and vibrated at $37^{\circ} \mathrm{C}$ by constant temperature oscillator for $30 \mathrm{~min}$. The amount of liquid was about 5 times of the size of bone granules. Next, $0.25 \%$ trypsin/EDTA was added, and specimens were digested with trypsin of a final concentration of $0.1 \%$ at $37^{\circ} \mathrm{C}$ for $5 \mathrm{~min}$ by constant temperature oscillator. Cell suspensions were collected by filtering out fat and bone granules with a 150 $\mu \mathrm{m}$ cell sieve, and then centrifuged at 1,500 $\mathrm{r} / \mathrm{min}$ for $3 \mathrm{~min}$ at room temperature. After centrifugation, a small cell mass was observed at the bottom of the tube, which was divided into two layers, the upper layer was mainly consisted of red cells and the lower layer was largely consisted of vascular endothelial cells. The cells were first suspended in a gelatin free T75 cell culture flask (Corning, NY) with DMEM-LG medium. One hour later, the culture medium was collected, then centrifuged again. After that, the cells were resuspended in $5 \mathrm{~mL}$ complete endothelial cell culture medium $(40 \mathrm{u} / \mathrm{mL}$ heparin, $100 \mathrm{u} / \mathrm{mL}$ penicillin, $100 \mathrm{ug} / \mathrm{mL}$ streptomycin, 20\% FCS, ECGM containing M199 medium, 10 ng/mL VEGF). Cells were counted in a haemocytometer, and the cell density was adjusted to $1,105 / \mathrm{mL}$ and plated on culture flasks coated with $2 \%$ gelatin.

\section{Culture of cells}

The cultured cells were maintained in $5 \% \mathrm{CO}_{2}$ at $37{ }^{\circ} \mathrm{C}$ with medium changed every 2-3 days. At 6-8 days, when the primary culture had grown to approximately $80 \%$ confluence, cells were detached by digestion with $0.25 \%$ trypsin/EDTA for $5 \mathrm{~min}$, and then seeded to new flasks at a split ratio of 1:3. Afterwards, culture medium was changed every 2-3 days and passages were performed when cells reached $80 \%$ confluence. 


\section{Morphological observation}

BMECs were plated in a T75 cell culture flask (Corning, NY, USA) and once grown to $80 \%$ confluence, cells were observed under inverted phase contrast microscope. Then these cells were washed by PBS for three times and collected by using a cytology brush. After centrifugation, cell mass was fixed with $2.5 \%$ glutaraldehyde for 24 hours at $4{ }^{\circ} \mathrm{C}$, soaked in $1 \%$ osmic acid for one hour, dehydrated with gradient acetone and then embedded by EPon- 812 . After the ultrathin sections were made and processed with double staining method of lead and uranium, they were observed under transmission electron microscope.

\section{Immunofluorescent staining}

Cells were cultured on gelatin-coated glass coverslips and left for 2-3 days to reach confluence. The coverslips were washed with PBS three times and then fixed with $4 \%$ paraformaldehyde at room temperature for $15 \mathrm{~min}$. After washing with PBS three times, cells were treated with $0.5 \%$ Triton X-100 for $20 \mathrm{~min}$ and then incubated in 10\% FBS for $30 \mathrm{~min}$ at room temperature. Subsequently, cells were incubated with the primary antibodies at $4{ }^{\circ} \mathrm{C}$ overnight in a wet box. Afterwards, coverslips were washed with PBS three times, and incubated with the secondary antibodies and Hoechst 33342 for $1 \mathrm{~h}$ at $20-37^{\circ} \mathrm{C}$, and then washed three times with PBS. Finally, the cells were sealed with mounting medium containing fluorescent preservative mounting agent, and viewed with a fluorescence microscope.

\section{Results}

\section{Morphology observation}

The primary cells were spindle shaped, grew in a manner of colony. After 24 hours, the cells were observed under inverted microscope. The cells of the patients less than 60 years old were evenly distributed, and each field $(\times 100)$ contained $50-100$ cells. The number of cells per field decreased with age, and only $2-10$ cells were seen per field for patients over the age of 85 . In general, the primary cultured HSVECs underwent 3 passages, and went through 2 continuous subculture cycles in total. After 3-4 days of culture, adherent cells were observed in many areas of the culture flasks, evenly distributed, and inconsistent in size and shape. The number of cells was inversely correlated with the age of patients in primary culture. Following $7-8$ days of primary culture, the cells grew against the wall of flask, formed a monolayer after fusion, arranged in fascicular, whorled, and contact inhibition appeared significantly (Figure 1A). Most of the cells appeared short spindle, polygon shaped and cobblestone-like morphology (Figure 1B). Under transmission electron microscope, these cells were in polygon shape and the surface was covered by microvilli formed by cytoplasm bulge, with large, ovular nuclei of obvious nucleoli. Cytoplasm was rich in mitochondria, ribosomes, rough endoplasmic reticulum, Golgi complexes and other organelles (Figure 1C).

\section{Immunofluorescence}

The surface markers of HSVECs were detected via immunofluorescence. The percentage of $\mathrm{vWF}$ positive cells was quantified by computed the mean from 3 randomly selected fields, and the results showed that about $100 \%$ of the cells were vWF positive. Specific bright yellow green fluorescence was observed in the plasmas and the nucleus was stained blue with Hoechst 33342 . VCAM-1 positive percentage was close to $100 \%$. The cells showed yellow green immunofluorescence, indicating that they expressed VCAM-1. CD31 antigens cells showed green fluorescence, and the percentage of which was about $100 \%$. The percentage of cells positive for Emcn was close to $100 \%$, and the majority of cells had moderate or high intensity of the yellow green fluorescence. The control groups were negative (Figure 2). The results of immunofluorescence of the isolated cells revealed positive expression of $\mathrm{vWF}$, VCAM-1, CD31 and Emcn, which indicated the cells were HSVECs.

\section{Discussion}

Bone microvascular system is an important part of bone microenvironment. Bone microvessels not only support metabolic needs of various kinds of cells in bone, but also have secretory function which plays a significant regulatory role in the metabolism of local bone tissue. The bone microvascular structure is similar to coral shape, and its capillaries, partially dilated, tortuous, and intertwined, are a complete three-dimensional frame formed by the connection of the thin-walled vascular sinus (28). Microcirculation dysfunction will affect new bone formation, bone resorption, nutrient transport and the balance of microenvironment in the bone. The blood vessels in bone tissue play an important role in the maintenance of bone mineral density, and decreased vascular support 

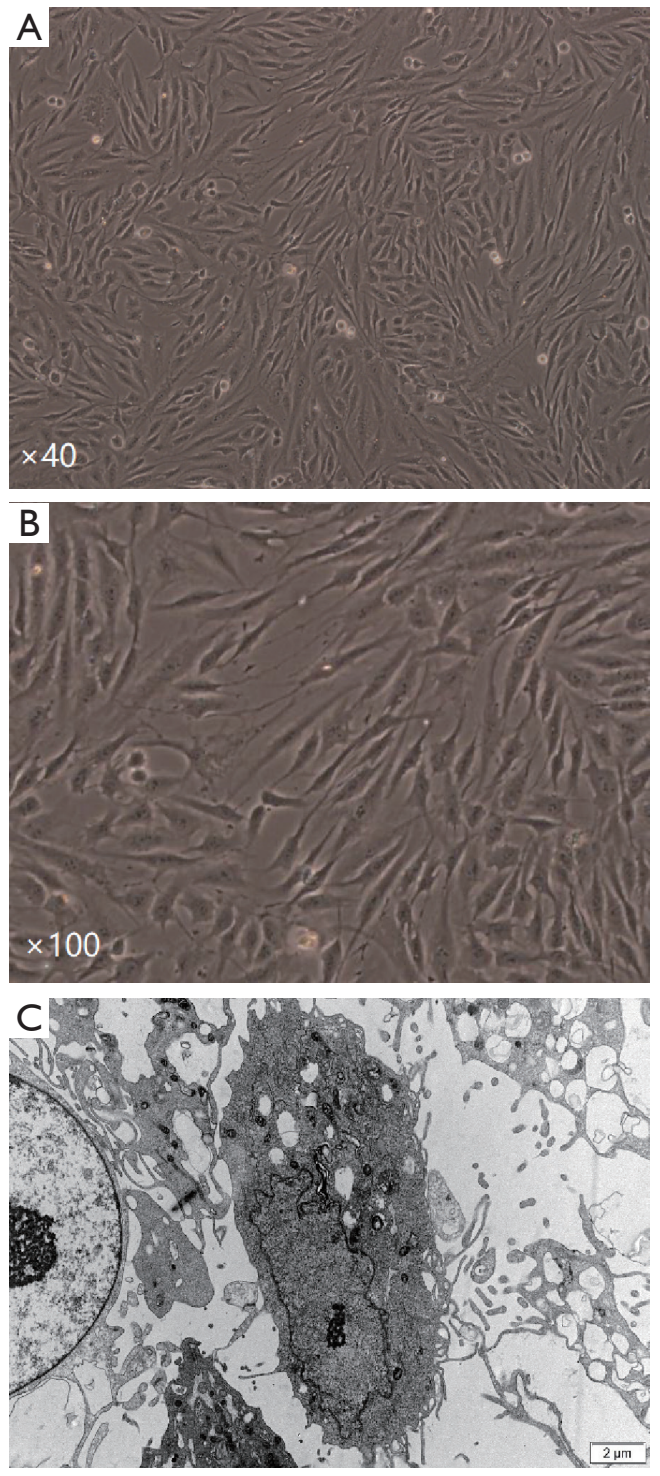

Figure 1 The HSVEC grew against the wall of flask, formed a monolayer after fusion, arranged in fascicular, whorled, and contact inhibition appeared significantly (A, inverted phase contrast microscope, $\times 40$ ). Most of the cells appeared short spindle, polygon shaped and cobblestone-like morphology (B, inverted phase contrast microscope, $\times 100)$. Under transmission electron microscope, these cells were in polygon shape and the surface was covered by microvilli formed by cytoplasm bulge, with large, ovular nuclei of obvious nucleoli. Cytoplasm was rich in mitochondria, ribosomes, rough endoplasmic reticulum, Golgi complexes and other organelles $(\mathrm{C}$, transmission electron microscope, stained with lead acetate, $\times 12 \mathrm{k}$ ). HSVEC, $\mathrm{H}$ subtype vascular endothelial cell. can result in increased bone loss (29). The typical blood supply of bone tissue (such as long tubular bones) is mainly provided by three types of blood vessels (feeding artery, metaphyseal-epoepiphyseal artery, and periosteal artery) (18). These blood vessels are all attached with vascular endothelial cells. However, how the end of the blood supply affects bone formation has become the focus of current research. In 2014, Kusumbe et al. $(18,30)$ firstly proposed that there is a coupling relationship between the growth of blood vessel terminals in bone and bone formation. Animal experiments found that most of the capillaries in the end of the artery in the bone terminate at the metaphysis near the growth plate, and a few in the endometrium; the blood vessels in the skeleton of mice can be divided into type $\mathrm{H}$ blood vessels and type $\mathrm{L}$ blood vessels. A large number of osteoprogenitor cells and osteoblasts clustered around type $\mathrm{H}$ blood vessels. $\mathrm{H}$ subtype blood vessels are rich in endothelial cells, which secrete Noggin to maintain the function of osteoblasts and chondrocytes which are responsible for bone formation. HSVECs are a special type of vascular endothelial cells. And these cells, in return, secrete VEGF to support angiogenesis. The type $\mathrm{H}$ vessels have been identified as a sensitive marker for bone loss in human bone structures, and changes in them represent an early event for bone deterioration (19).

Several researchers have isolated and cultured vascular endothelial cells from the bone marrow of the ilium and studied the hematopoietic function of bone marrow in vitro (23-25). These endothelial cells were not endothelial cells in the vessel walls of the bone, but a kind of bone marrow stromal cells, its function may be different from traditional BMECs. Our institution $(26,27,31)$ has successfully isolated the BMECs from the femoral head to study ONFH. Until recently, most experiments with vascular endothelial cells have been conducted with unsorted cells. On the basis of these studies, we isolated the HSVECs which would be an ideal cell source for the later study on ONFH. Our study is the first report that obtaining highly pure HSVECs of human femoral head by using enzymatic digestion and purification of selective medium. This study draws on the experience of the separation method of Lu et al. (27), using $0.2 \%$ collagenase I to digest the tissue for $30 \mathrm{~min}$ at $37^{\circ} \mathrm{C}$, which is an essential procedure of obtaining enough endothelial cells and removing other tissues. Using 


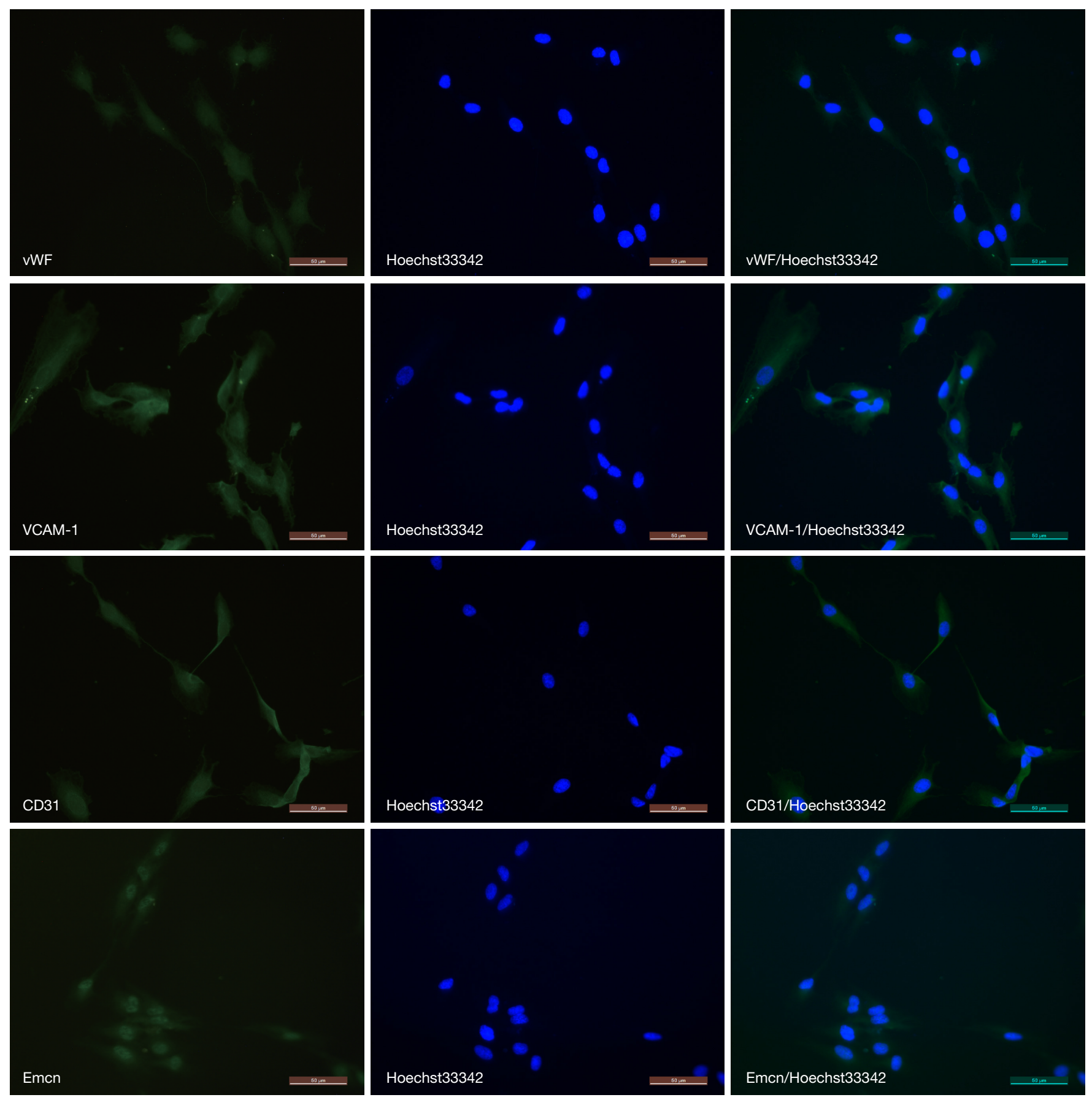

Figure 2 The HSVECs expressing vWF, VCAM-1, CD31 and Emcn were identified by immunofluorescence. vWF, von Willebrand factor; HSVEC, H subtype vascular endothelial cell.

complete endothelial cell culture medium containing endothelial cell growth factor and heparin to stimulate the growth of endothelial cells, we can effectively remove fibroblasts and other impurities. vWF, VCAM-1 and CD31 are routinely used for the definition of human vascular endothelial cells. vWF is a large multimeric glycoprotein that showed an obvious effect on hemostasis and thrombus formation (32), and normally synthesized by vascular 
endothelial cells and megakaryocytes (33). The type $\mathrm{H}$ vessel has been identified in human bone and is positive for CD31 and Emcn on the range of organs and tissues demonstrated by immunohistochemistry (18). CD31, expressing in endothelial cell and platelet in high level, is commonly used to identify vascular endothelial cells in recent years (34). Emcn was identified as an endothelial sialomucin expressed on the surface of endothelial cells along the vascular tree, and it serves as a specific marker in the study of endothelial cells $(35,36)$.

The co-localization of CD31 and Emcn indicated that the cultured cells were HSVECs. Moreover, it was observed that HSVECs exhibited the typical morphology of vascular endothelial cells during culture. Wang et al. (19) found that type $\mathrm{H}$ vessels was enriched in the proximal femur near the greater trochanter. This study successfully obtained large amount of purity HSVECs, and proved that human femoral head contains a high percentage of type $\mathrm{H}$ vessels, and CD31 and Emcn can be used as reliable markers for the prospective isolation of HSVECs. We also found that the number of HSVECs after 24 hours of primary culture was directly related to the age of the patients. It is easier to culture more HSVECs from the bone tissue of young patients. Due to the rapidly increase of aging population, the number of patients with osteoporosis has been increasing annually (37). Because of the close correlation between $\mathrm{H}$ type vessel and bone loss, the research on HSVECs will also provide a new research direction for the treatment of osteoporosis.

This study has some limitations. The method of differential adhesion was used to eliminate the contamination of fibroblasts, but there were still chances of contamination of fibroblasts in primary culture. As a secondary precaution, using complete endothelial cell culture medium helps to eliminate the possible contamination of fibroblasts. Moreover, it is possible that the cultured HSVECs may behave differently from freshly isolated HSVECs. This problem may exist in all in vitro cultured cells. We tried to complete co-localization immunofluorescent staining with different markers for HSVECs, but the effect of the co-localization is not always satisfactory. Probably the immunofluorescence graphs of different markers influence each other, which makes the graph unclear. We will continue to explore the colocalization technology in the future. However, the CD31 and Emcn are highly expressed, respectively, in the same group of the cultured cells by immunofluorescence staining, which is confirmed to be HSVECs. Considering that there have already been some detailed papers on the function and its related mechanism of $\mathrm{H}$ subtype blood vessels, this study did not involve this aspect of HSVECs. However, the detailed function of human HSVECs will be the direction of our study in future.

\section{Conclusions}

In summary, we describe a simple and effective experimental verification for isolation of HSVECs from the human femoral heads. These purified cells may become an important tool for the studies on ONFH, osteoporosis and other bone diseases.

\section{Acknowledgments}

Thanks are due to Anna Peltola for English Language Editing.

Funding: This study was supported by the National Natural Science Foundation of China $(81802224,81672236$, 81871830), The Capital's Funds for Health Improvement and Research (CFH2018-4-40611), Beijing Municipal Science \& Technology Commission No. Z181100001718058, the Fundamental Research Funds for the Central Universities (3332018168), Graduate Innovation Foundation of Peking Union Medical College (2017-1002-2-26), Ningxia Natural Science Foundation (2020AAC03337) and Beijing Natural Science Foundation (7182146).

\section{Footnote}

Data Sharing Statement: Available at http://dx.doi. org/10.21037/apm-20-121

Conflicts of Interest: All authors have completed the ICMJE uniform disclosure form (available at http://dx.doi. org/10.21037/apm-20-121). The authors have no conflicts of interest to declare.

Ethical Statement: The authors are accountable for all aspects of the work in ensuring that questions related to the accuracy or integrity of any part of the work are appropriately investigated and resolved. The trial was conducted in accordance with the Declaration of Helsinki (as revised in 2013). The study was approved by institutional ethics board of China-Japan Friendship Hospital (No. 2016-BZR-2) and informed consent was taken from all the patients. 
Open Access Statement: This is an Open Access article distributed in accordance with the Creative Commons Attribution-NonCommercial-NoDerivs 4.0 International License (CC BY-NC-ND 4.0), which permits the noncommercial replication and distribution of the article with the strict proviso that no changes or edits are made and the original work is properly cited (including links to both the formal publication through the relevant DOI and the license). See: https://creativecommons.org/licenses/by-nc-nd/4.0/.

\section{References}

1. Mont MA, Jones LC, Hungerford DS. Nontraumatic osteonecrosis of the femoral head: ten years later. J Bone Joint Surg Am 2006;88:1117-32.

2. Drescher W, Pufe T, Smeets R, et al. Avascular necrosis of the hip-diagnosis and treatment. Z Orthop Unfall 2011;149:231-40.

3. Wang T, Teng S, Zhang Y, et al. Role of mesenchymal stem cells on differentiation in steroid-induced avascular necrosis of the femoral head. Exp Ther Med 2017;13:669-75.

4. Assouline-Dayan Y, Chang C, Greenspan A, et al. Pathogenesis and natural history of osteonecrosis. Semin Arthritis Rheum 2002;32:94-124.

5. Saito $\mathrm{S}$, Inoue A, Ono K. Intramedullary haemorrhage as a possible cause of avascular necrosis of the femoral head: the histology of 16 femoral heads at the silent stage. J Bone Joint Surg Br 1987;69:346-51.

6. Arlet J. Nontraumatic avascular necrosis of the femoral head. Past, present, and future. Clin Orthop Relat Res 1992;(277):12-21.

7. Mont MA, Hungerford DS. Nontraumatic avascular necrosis of the femoral head. J Bone Joint Surg 1995;77:459-74.

8. Lavernia CJ, Sierra RJ, Grieco FR. Osteonecrosis of the femoral head. J Am Acad Orthop Surg 1999;7:250-61.

9. Starklint H, Lausten GS, Arnoldi CC. Microvascular obstruction in avascular necrosis. Immunohistochemistry of 14 femoral heads. Acta Orthop Scand 1995;66:9-12.

10. Kerachian MA, Harvey EJ, Cournoyer D, et al. Avascular necrosis of the femoral head: vascular hypotheses. Endothelium 2006;13:237-44.

11. Brandi ML, Collin-Osdoby P. Vascular biology and the skeleton. J Bone Miner Res 2006;21:183-92.

12. Schipani E, Wu C, Rankin EB, et al. Regulation of bone marrow angiogenesis by osteoblasts during bone development and homeostasis. Front Endocrinol
(Lausanne) 2013;4:85

13. Glowacki J. Angiogenesis in fracture repair. Clin Orthop Relat Res 1998;(355 Suppl):S82-9.

14. Tomlinson RE, Silva MJ. Skeletal blood flow in bone repair and maintenance. Bone Res 2013;1:311-22.

15. Saran U, Gemini Piperni S, Chatterjee S. Role of angiogenesis in bone repair. Arch Biochem Biophys 2014;561:109-17.

16. Maes C, Kobayashi T, Selig MK, et al. Osteoblast precursors, but not mature osteoblasts, move into developing and fractured bones along with invading blood vessels. Dev Cell 2010;19:329-44.

17. Garcia P, Pieruschka A, Klein M, et al. Temporal and spatial vascularization patterns of unions and nonunions: role of vascular endothelial growth factor and bone morphogenetic proteins, J Bone Joint Surg Am 2012;94:49-58.

18. Kusumbe AP, Ramasamy SK, Adams RH. Coupling of angiogenesis and osteogenesis by a specific vessel subtype in bone. Nature 2014;507:323-8.

19. Wang L, Zhou F, Zhang P, et al. Human type $H$ vessels are a sensitive biomarker of bone mass. Cell Death Dis 2017;8:e2760.

20. Xie H, Cui Z, Wang L, et al. PDGF-BB secreted by preosteoclasts induces angiogenesis during coupling with osteogenesis. Nat Med 2014;20:1270-8.

21. Pouya F, Kerachian MA. Avascular Necrosis of the Femoral Head: Are Any Genes Involved? Arch Bone Jt Surg 2015;3:149-55.

22. Ding H, Gao YS, Hu C, et al. HIF-1alpha transgenic bone marrow cells can promote tissue repair in cases of corticosteroid-induced osteonecrosis of the femoral head in rabbits. PLoS One 2013;8:e63628.

23. Rafii S, Shapiro F, Rimarachin J, et al. Isolation and characterization of human bone marrow microvascular endothelial cells: hematopoietic progenitor cell adhesion. Blood 1994;84:10-9.

24. Candal FJ, Rafii S, Parker JT, et al. BEMC-1: human bone marrow microvascular endothelial cell line with primary cell characteristics. Microvasc Res 1996;52:221-34.

25. Rafii S. Shapiro F. Pettengell R, et al. Human bone marrow microvascular endothelial cells support longterm proliferation and differentiation of myeloid and megakaryocytic progenitors. Blood 1995;86:3353-63.

26. Yang Y, Lou J, Li Z, et al. Effect of glucocorticoid on production of reactive oxygen species in bone microvascular endothelial cells. Zhongguo Xiu Fu Chong Jian Wai Ke Za Zhi 2011;25:533-7. 
27. Lu YF, Yu Q, Guo W, et al. A method for isolated culture of bone microvascular endothelial cells of human femoral head. Zhongguo Gu Shang 2014;27:843-7.

28. Wang BL, Li ZR. Progress in the study of microvascular endothelial cells in bone marrow microenvironment. Chinese Microcirculation 2006;10:451-3.

29. Vogt MT, Cauley JA, Kuller LH, et al. Bone mineral density and blood flow to the lower extremities: the study of osteoporotic fractures. J Bone Miner Res 1997;12:283-9.

30. Kusumbe AP, Adams RH. Osteoclast progenitors promote bone vascularization and osteogenesis. Nat Med 2014;20:1238-40.

31. Wang BL, Li ZR, Lou JN, et al. Protection of Human Bone Microvascular Endothelial Cells from Glucocorticosteroid Induced Cell Injury by Icariin in vitro. Chinese Microcirculation 2009;13:n461-4.

32. Sadler JE. Von Willebrand factor, ADAMTS13, and thrombotic thrombocytopenic purpura. Blood

Cite this article as: Gao F, Mao T, Zhang Q, Han J, Sun W, Li Z. H subtype vascular endothelial cells in human femoral head: an experimental verification. Ann Palliat Med 2020;9(4):14971505. doi: 10.21037/apm-20-121
2008;112:11-8.

33. Zheng XL. ADAMTS13 and von Willebrand factor in thrombotic thrombocytopenic purpura. Annu Rev Med 2015;66:211-25.

34. Kacemi A, Challier JC, Galtier M, et al. Culture of endothelial cells from human placental microvessels. Cell Tissue Res 1996;283:183-90.

35. Kuhn A, Brachtendorf G, Kurth F, et al. Expression of endomucin, a novel endothelial sialomucin, in normal and diseased human skin. J Invest Dermatol 2002;119:1388-93.

36. dela Paz NG, D'Amore PA. Arte rial versus venous endothelial cells. Cell Tissue Res 2009;335:5-16.

37. Yoshimura N, Muraki S, Oka H, et al. Prevalence of knee osteoarthritis, lumbar spondylosis and osteoporosis in Japanese men and women: the research on osteoarthritis/ osteoporosis against disability study. J Bone Miner Metab 2009;27:620-8. 